\title{
Templates for stellar mass black holes falling into supermassive black holes
}

\author{
B S Sathyaprakash ${ }^{1}$ and B F Schutz ${ }^{1,2}$ \\ ${ }^{1}$ Department of Physics and Astronomy, Cardiff University, Cardiff, UK \\ 2 Albert-Einstein Institute, Golm, Germany
}

Received 10 December 2002, in final form 14 January 2003

Published 29 April 2003

Online at stacks.iop.org/CQG/20/S209

\begin{abstract}
The spin-modulated gravitational wave signals, which we shall call smirches, emitted by stellar mass black holes, tumbling and inspiralling into massive black holes, have extremely complicated shapes. Tracking these signals with the aid of pattern matching techniques, such as Wiener filtering, is likely to be computationally an impossible exercise. In this paper we propose using a mixture of optimal and non-optimal methods to create a search hierarchy to ease the computational burden. Furthermore, by employing the method of principal components (also known as singular value decomposition) we explicitly demonstrate that the effective dimensionality of the search parameter space of smirches is likely to be just 3 or 4 , much smaller than what has hitherto been thought to be about 9 or 10 . This result, based on a limited study of the parameter space, should be confirmed by a more exhaustive study over the parameter space as well as Monte Carlo simulations to test the predictions made in this paper.
\end{abstract}

PACS numbers: $04.80 . \mathrm{Nn}$, 95.55.Ym, 95.75.Pq, 97.80.Af

\section{Introduction}

A stellar mass compact object, either a neutron star or a black hole, in tight orbit around a massive Kerr black hole will tumble around the massive companion due to strong spin-orbit coupling. For small mass ratios the stellar mass companion slowly inspirals into the massive hole, the duration of inspiral increasing inversely as the mass ratio. Gravitational waves emitted in the process of adiabatic inspiral encode the structure of the spacetime geometry around the hole thereby allowing us to test the unique theorems of black-hole spacetimes [1]. However, decoding the information carried by the waves would require an accurate knowledge of how the emitted waves depend on the parameters of the system and fit the data with the expected signal. There are problems with both of these aspects: on one hand we do not currently have an accurate knowledge of the signal although over the next decade the situation is likely to 
have improved. On the other hand, the problem of fitting is unlikely to go away as the number template waveforms required to decode the useful information might be formidably large. This is because the dimensionality of the parameter space is quite large. Indeed, in the generic case of a binary consisting of two spinning black holes, there are potentially 17 parameters to fit: the direction and distance to the source, two masses, three vectors corresponding to the initial values of the orbital angular momentum and two spin angular momenta, the eccentricity of the orbit, the instant when the orbital frequency reaches a fiducial value and the phase of the signal at that instant.

Even though the time structure of the signal depends on 17 parameters, different parameter sets may not always produce distinct waveforms. In other words, the effective dimensionality of the parameter space for the purpose of fitting might be much smaller. For instance, we know in the case of non-spinning binaries that out of potentially eight parameters - namely, the direction to the source and its distance from the Earth, two masses, an initial magnitude of the angular momentum, a fiducial time and phase when the signal reaches a certain frequencythere is effectively only one parameter with which to fit the data. This is the so-called chirp mass, which is a certain combination of the two masses of the component bodies. The detector response has no time-varying dependence on the source direction since the signal lasts for a very short duration (minutes, at most) and therefore does not suffer any modulation due to the motion of the antenna that could have been useful in deciphering the source direction; the initial magnitude of the angular momentum only sets an initial frequency of observation that is chosen by hand; the fiducial time and phase can be easily determined by the use of the fast Fourier transform and analytically, respectively; and the signal's phasing, which is important in accomplishing a good fit, depends, at the dominant post-Newtonian order, only on the chirp mass. Although the degeneracy in component masses is resolved when higher order postNewtonian terms are included in the phasing, it turns out that the number of 'independent' shapes does not increase significantly while including a second mass parameter.

An analysis similar to the non-spinning case is required in the case of spinning black-hole binaries. In this paper, we discuss the method of principal components [2] that can be used to determine the effective dimensionality of the search space. We will then apply it to the case of a test mass falling into a massive black hole. We sample the parameter space at a few crucial points to get an idea of the number of principal components on which the signal shape depends, thereby obtaining an estimate of how large the parameter space could be.

Since we expect the number of templates to be too large, it will not be possible to employ matched filtering on an entire year's worth of data. Rather, the idea is to employ a technique, such as time-frequency analysis, that makes little use of the signal shape in capturing a signal, albeit with a lower detection probability. Such an analysis might help to first get a rough idea about the signal parameters. One could then employ matched filtering on, say, a week's worth of data and refine the estimation of parameters. Based on these refined parameters one finally employs the search on a year's worth of data but in a small region of the parameter space. In other words, we are advocating a multi-step hierarchical search involving different analysis techniques. In such a scheme it would only be necessary to estimate the number of templates required in the most compute-intensive stage of the search hierarchy, namely in the first stage of matched filtering. It is best to begin the search from a point where the parameters can be nailed down most easily, namely by employing matched filtering on a week's worth of data. (At this stage a signal-to-noise ratio of about 5-10 can be expected for mergers taking place at a distance of about a few Gpc.) This assumption somewhat simplifies our calculation as we can neglect the motion of LISA relative to the source during this period and assume that the detector is fixed relative to the source. Strictly speaking, this is not a valid assumption but it is unlikely to change the qualitative conclusions reported in this work. 


\section{Counting the number of templates-a rough estimate}

To count the number of templates we must first understand how the signal evolves as a function of time. To this end we shall use the best post-Newtonian order currently known but ignore eccentricity. It turns out that at this order the signal already has many modulations including spin-orbit and spin-spin couplings. Therefore, the waveform should be quite suitable for counting template numbers although it may not be good enough for the purpose of fitting.

\subsection{Evolution of the orbit}

Following [3] we shall use a coordinate system adapted to LISA. In the post-Newtonian approximation the evolution of a binary system comprising two bodies of masses $m_{1}$ and $m_{2}$, spins $\mathbf{S}_{1}=\left(s_{1}, \theta_{1}, \varphi_{1}\right)$ and $\mathbf{S}_{2}=\left(s_{2}, \theta_{2}, \varphi_{2}\right)$, orbital angular momentum $\mathbf{L}=\left(L, \theta_{0}, \varphi_{0}\right)$, is governed by a set of differential equations given by

$\dot{\mathbf{L}}=\left[\left(\frac{4 m_{1}+3 m_{2}}{2 m_{1} m^{3}}-\frac{3 \mathbf{S}_{2} \cdot \mathbf{L}}{2 L^{2} m^{3}}\right) \mathbf{S}_{1}+\left(\frac{4 m_{2}+3 m_{1}}{2 m_{2} m^{3}}-\frac{3}{2} \frac{\mathbf{S}_{1} \cdot \mathbf{L}}{L^{2} m^{3}}\right) \mathbf{S}_{2}\right] \mathbf{L} v^{6}-\frac{32 \eta^{2} m}{5 L} \mathbf{L} v^{7}$,

$\dot{\mathbf{S}}_{1}=\left[\left(\frac{4 m_{1}+3 m_{2}}{2 m_{1} m^{3}}-\frac{3 \mathbf{S}_{2} \cdot \mathbf{L}}{2 L^{2} m^{3}}\right) \mathbf{L} \times \mathbf{S}_{1}+\frac{\mathbf{S}_{2} \times \mathbf{S}_{1}}{2 m^{3}}\right] v^{6}$,

$\dot{\mathbf{S}}_{2}=\left[\left(\frac{4 m_{2}+3 m_{1}}{2 m_{2} m^{3}}-\frac{3 \mathbf{S}_{1} \cdot \mathbf{L}}{2 L^{2} m^{3}}\right) \mathbf{L} \times \mathbf{S}_{2}+\frac{\mathbf{S}_{1} \times \mathbf{S}_{2}}{2 m^{3}}\right] v^{6}$.

Here $v=(\pi m f)^{1 / 3}$, where $f$ is the gravitational wave frequency, is the post-Newtonian velocity parameter, an overdot denotes the time derivative, $m=m_{1}+m_{2}$ is the total mass, and $\eta=m_{1} m_{2} / m^{2}$ is the (symmetric) mass ratio. In the evolution of the orbital angular momentum we have included the lowest order dissipative term (the second term containing $v^{7}$ in equation (1)) while keeping the non-dissipative modulation effects caused by spin-orbit and spin-spin couplings (the first term within square brackets containing $v^{6}$ in equation (1)). The spins evolve non-dissipatively but their orientations change due to spin-orbit and spinspin couplings. Though the non-dissipative terms are not responsible for gravitational wave emission, and therefore do not shrink the orbit, they cause the orbit to precess.

In the absence of spins the antenna observes the same polarization at all times; the amplitude and frequency of the signal both increase monotonically, giving rise to a chirping signal. Precession of the orbit and spins cause modulations in the amplitude and phase of the signal, and smear the signal's energy spectrum over a wide band. The smearing is particularly dramatic when one of the bodies is much lighter than the other, such as the inspiral of a stellar mass compact object into a massive black hole. In this case the orbit would undergo many precessions before the small object plunges into the hole smearing the signal into hundreds of frequency bins as opposed to a few frequency bins if the signal were a simple chirp. For this reason we shall call the spin-modulated ( $\mathrm{sm}$ ) chirp, a smirch (an anagram of sm and chir).

\subsection{The waveform}

The strain $h(t)$ produced by a smirch in LISA is given by

$$
h(t)=-A(t) \cos [\Phi(t)+\varphi(t)],
$$

where $A(t)$ is the precession-modulated amplitude of the signal, $\Phi(t)$ is its post-Newtonian carrier phase that increases monotonically and $\varphi(t)$ is the polarization phase caused by the changing polarization of the wave relative to the antenna. (We have neglected the Thomas 
precession of the orbit which induces additional, but small, corrections in the phase.) For a source with position vector $\mathbf{N}=\left(D, \theta_{S}, \varphi_{S}\right)$ the amplitude is given by

$A(t)=\frac{2 \eta m v^{2}}{D}\left[\left(1+(\hat{\mathbf{L}} \cdot \hat{\mathbf{N}})^{2}\right)^{2} F_{+}^{2}\left(\theta_{S}, \varphi_{S}, \psi\right)+4(\hat{\mathbf{L}} \cdot \hat{\mathbf{N}})^{2} F_{\times}^{2}\left(\theta_{S}, \varphi_{S}, \psi\right)\right]^{1 / 2}$.

Here $\hat{\mathbf{L}}=\mathbf{L} / L, \hat{\mathbf{N}}=\mathbf{N} / D(D$ is the distance to the source $)$ and $\psi(t)$ is the precessionmodulated polarization angle given by

$$
\tan \psi(t)=\frac{\hat{\mathbf{L}}(t) \cdot \hat{\mathbf{z}}-(\hat{\mathbf{L}}(t) \cdot \hat{\mathbf{N}})(\hat{\mathbf{z}} \cdot \hat{\mathbf{N}})}{\hat{\mathbf{N}} \cdot(\hat{\mathbf{L}}(t) \times \hat{\mathbf{z}})} .
$$

Also, $F_{+}$and $F_{\times}$are the antenna beam pattern functions given by

$$
\begin{aligned}
& F_{+}\left(\theta_{S}, \varphi_{S}, \psi\right)=\frac{1}{2}\left(1+\cos ^{2} \theta_{S}\right) \cos 2 \phi_{S} \cos 2 \psi-\cos \theta_{S} \sin 2 \phi_{S} \sin 2 \psi, \\
& F_{\times}\left(\theta_{S}, \phi_{S}, \psi\right)=\frac{1}{2}\left(1+\cos ^{2} \theta_{S}\right) \cos 2 \phi_{S} \sin 2 \psi+\cos \theta_{S} \sin 2 \phi_{S} \sin 2 \psi
\end{aligned}
$$

Next, the polarization phase $\varphi(t)$ is

$$
\tan \varphi(t)=\frac{2 \hat{\mathbf{L}}(t) \cdot \hat{\mathbf{N}} F_{\times}\left(\theta_{S}, \varphi_{S}, \psi\right)}{\left[1+(\hat{\mathbf{L}}(t) \cdot \hat{\mathbf{N}})^{2}\right] F_{+}\left(\theta_{S}, \varphi_{S}, \psi\right)} .
$$

Finally, for the carrier phase we use the post-Newtonian expression, but without the spinorbit and spin-spin couplings. These spin couplings modify the carrier phase by amounts much smaller than the post-Newtonian effects so that neglecting them makes no appreciable difference to our main conclusions. To second post-Newtonian order the carrier phase is given by

$\Phi(t)=\frac{-2}{\eta \Theta^{5}}\left[1+\left(\frac{3715}{8064}+\frac{55}{96} \eta\right) \Theta^{2}-\frac{3 \pi}{4} \Theta^{3}+\left(\frac{9275495}{14450688}+\frac{284875}{258048} \eta+\frac{1855}{2048} \eta^{2}\right) \Theta^{4}\right]$,

where $\Theta=\left[\eta\left(t_{C}-t\right) /(5 m)\right]^{-1 / 8}, t_{C}$ being the time at which the two stars merge together and the gravitational wave frequency formally diverges.

\subsection{A rough count of templates}

From the foregoing equations we can get an idea of the number of parameters that may be needed in a search. The system we are interested in is that of a stellar mass black hole falling into a supermassive black hole wherein the mass ratio could be as low as $10^{-5}$. Consequently, the spin of the stellar mass hole, being proportional to the square of its mass, would be negligible compared to the spin of the massive hole. Further, we do not have to specifically search for the distance to the binary nor for the fiducial time and phase when the frequency of the emitted gravitational wave reaches a certain value as they can all be measured without incurring significant computational costs. Moreover, since we are focussing on quasi-circular orbits (i.e., zero eccentricity) and only those systems that merge within a week of observation (i.e., after about 1000 orbital time scales), the number of parameters in our search is down from 17 to 9 . These are the initial direction of the orbital angular momentum (two angles), the spin of the massive black hole (one magnitude and two angles), the masses of the two bodies (two numbers) and the direction to the source (two angles).

As mentioned in the introduction, we do not expect both masses to be important but only a combination-the chirp mass $\mathcal{M}=\eta^{3 / 5} \mathrm{~m}$. The azimuth angles are normally not important in a search but co-latitudes would lead to modulations that cannot be easily searched for. Consequently, out of the six angles we expect only three to be important. (Note that due 
to different orientations of the binary and antenna these angles get mixed up and therefore azimuth and co-latitudes are not well-defined. However, we expect certain combinations of these angles to play the roles of azimuth and co-latitude. Our claim is that three of these combinations would be important in a search and the other three not so important.) In addition, we expect the spin magnitude of the hole to be a search parameter although this would be important only when $s_{1}$ is close to 1 . As a result the number of search parameters would be five or less. Assuming that we have to search for five parameters in all and that each parameter requires as many templates as the number of cycles, we get a rough count of $\left(10^{3}\right)^{5}=10^{15}$ templates. We shall see below that this is a rather large overestimate. The number of search parameters needs to be determined by a more rigorous and quantitative analysis which we take up in the next two sections.

\section{Covariance matrix, correlation coefficients and principal components}

Any measurement process is disturbed by a background perturbation or noise which causes systematic and/or random errors in the measured quantities. In general, the errors get smaller at greater signal-to-noise ratios. We review the theory behind estimating errors involved in a measurement process.

For a signal buried in a Gaussian background and detected using matched filtering, the covariance matrix $\Sigma_{k l}$ is a symmetric matrix whose diagonal elements are the variances in the measurement of various parameters and the off-diagonal elements are the covariances between them. The covariance matrix is itself the inverse of the information matrix, or the metric, $g_{m n}$. Let us suppose the signal $h\left(t ; \lambda_{k}\right)$ depends on $p$ parameters $\lambda_{k}, k=1, \ldots, p$. The information matrix is defined by

$$
g_{m n}=\left\langle\partial_{m} h, \partial_{n} h\right\rangle, \quad \Sigma_{m n}=\left[g^{-1}\right]_{m n},
$$

where for any two functions $a(t)$ and $b(t)$ (and Fourier transforms $\tilde{a}(f)$ and $\tilde{b}(f)$ ), their scalar product $\langle a, b\rangle$ is defined as

$$
\langle a, b\rangle=2 \int_{f_{\text {low }}}^{f_{\text {high }}} \frac{\mathrm{d} f}{S_{h}(f)}\left[\tilde{a}(f) \tilde{b}^{*}(f)+\tilde{a}^{*}(f) \tilde{b}(f)\right],
$$

where $S_{h}(f)$ is the (real) one-sided noise spectral density of the antenna and $f_{\text {low }}$ and $f_{\text {high }}$ are some appropriately defined lower and upper frequency cut-offs depending on the signal and the antenna responses.

Starting from the covariance matrix one defines the matrix of correlation coefficients $C_{k l}$ in the following manner:

$$
\begin{aligned}
C_{k l} & =\sqrt{\Sigma_{k l}}, \quad \text { if } \quad k=l, \\
& =\frac{\Sigma_{k l}}{\sqrt{\Sigma_{k k} \Sigma_{l l}}}, \quad \text { if } \quad k \neq l .
\end{aligned}
$$

As is obvious the diagonal element $C_{k k}$ of the matrix of correlation coefficients is the (onesigma) standard deviation in the measurement of the parameter $\lambda_{k}$, and the $k l$-off-diagonal element $C_{k l}$ is the normalized covariance between the parameters $\lambda_{k}$ and $\lambda_{l}$. The correlation coefficients $C_{k l}$ take values in the range $[-1,1]$. A correlation coefficient $C_{k l}$ close to 1 (or -1 ) indicates that the parameters $\lambda_{k}$ and $\lambda_{l}$ are perfectly correlated (or anti-correlated) with each other. If on the other hand the value is close to zero, then there is little correlation between the two parameters. When the two parameters have a correlation coefficient close to \pm 1 , then varying one of the parameters is equivalent to varying the other. In other words one does not produce two independent signal shapes by varying both the parameters, varying only one of 
them should be sufficient. However, when the two parameters are not correlated, then varying one of them produces a distinct shape as compared to varying the other. Therefore, we can look at the matrix of correlation coefficients to determine how many parameters are really independent.

Finally, we can diagonalize the covariance matrix to find the principal components. On diagonalization one can (linearly) transform the parameter set $\lambda_{k}$ to a new set $\mu_{k}$ effectively removing in the process all the covariances between the parameters. The principal components of the signal are those transformed parameters that correspond to the largest diagonal elements. Since we have (by construction) a symmetric matrix the eigenvalues are all real. Moreover, since the covariance matrix is the inverse of the metric the principal components are the inverses of the eigenvalues of the metric. Before we embark upon a discussion of the results let us look at two simple examples.

Our first example is a simple mathematical example. Consider a two-dimensional matrix $C_{k l}$. Suppose $C_{11}=C_{22}=1$ and $C_{12}=C_{21}=\epsilon<1$. The eigenvalues $\lambda_{ \pm}$of such a matrix are $\lambda_{ \pm}=1 \pm \epsilon$. If $\epsilon$ is close to zero then both the eigenvalues are roughly the same and both the parameters are equally important. If, however, $|\epsilon|$ is close to 1 then one of the eigenvalues is nearly zero making the corresponding transformed parameter unimportant. Thus, the closer the correlation coefficient is to \pm 1 , the smaller is the significance of the parameter.

As a second example let us consider a sinusoidal signal $h\left(t ; A, t_{0}, \varphi_{0}\right)=A \cos \left[\omega\left(t-t_{0}\right)+\right.$ $\left.\varphi_{0}\right]$. Here the parameters $A$ and $t_{0}$ are uncorrelated and so are $A$ and $\varphi_{0}$, while $t_{0}$ and $\varphi_{0}$ are perfectly anti-correlated. Increasing $\varphi_{0}$ is equivalent to decreasing $t_{0}$ but the signal has really only two independent parameters leading to two principal components.

\section{Correlation coefficients for smirches}

We now apply the formalism developed in the previous section to the gravitational wave signals produced by stellar mass black holes falling into supermassive black holes. We first compute the covariance matrix and then diagonalize it to obtain the principal components.

Let us first examine the matrix of correlation coefficients. In tables 1-3 we display the correlation coefficients of the smirches for three different values of the mass ratio, $\eta=10^{-3}, 10^{-4}, 10^{-5}$. We have also changed the orientations of the spin, orbital angular momentum and source direction from one example to the other to get an idea of how the correlation coefficients depend on the angular parameters. Tables 1-3 correspond to cases A, B and C, respectively; in all cases the total mass $m=10^{6} M_{\odot}, a_{1}=0.9$ and the initial orientation of the orbit is the same $\left(\theta_{L}, \varphi_{L}\right)=(\pi / 2, \pi / 3)$.

$$
\begin{array}{cccccc} 
& \eta & \theta_{1} & \varphi_{1} & \theta_{S} & \varphi_{S} \\
\text { Case A: } & 10^{-3} & \pi / 4 & \pi / 2 & \pi / 8 & \pi \\
\text { Case B: } & 10^{-4} & \pi / 3 & \pi / 4 & \pi / 2 & \pi / 3 \\
\text { Case C: } & 10^{-5} & \pi / 4 & \pi / 2 & \pi / 2 & \pi / 3
\end{array}
$$

The results reported here should be taken as a hint on what the general results are going to be but we cannot be absolutely certain about any of the results. We plan to report a full treatment of the problem with exhaustive Monte Carlo simulations to validate our results.

The diagonal elements in these tables are the expected standard deviations in the measurement of the corresponding parameters when the (amplitude) signal-to-noise ratio is 10. In the case of $m, \eta$ and $s_{1}$ we have quoted the relative error (namely, $\sigma_{k} / \lambda_{k}$ where $\sigma_{k}$ is the expected standard deviation and $\lambda_{k}$ is the true value of the parameter) while the error in angles is the absolute error expected in their measurements. Since the signal-to-noise 
Table 1. Correlation coefficients and errors in the estimation of parameters for a smirch corresponding to the following parameters: $m=10^{6} M_{\odot}, \eta=10^{-3}, a_{1}=0.9, \theta_{1}=\pi / 4, \varphi_{1}=$ $\pi / 2, \theta_{L}=\pi / 5, \varphi_{L}=\pi / 3, \theta_{S}=\pi / 8, \varphi_{S}=\pi$ and $n_{\mathrm{cyc}}=910$.

\begin{tabular}{|c|c|c|c|c|c|c|c|c|c|}
\hline$k$ & $m$ & $\eta$ & $a_{1}$ & $\theta_{1}$ & $\varphi_{1}$ & $\theta_{L}$ & $\varphi_{L}$ & $\theta_{S}$ & $\varphi_{S}$ \\
\hline$m$ & 0.0033 & & & & & & & & \\
\hline$\eta$ & -0.9592 & 0.0023 & & & & & & & \\
\hline$a_{1}$ & -0.9336 & 0.8775 & 0.0035 & & & & & & \\
\hline$\theta_{1}$ & 0.1516 & -0.1776 & -0.1301 & 0.3270 & & & & & \\
\hline$\varphi_{1}$ & -0.0275 & 0.0326 & 0.0262 & 0.7073 & 1.8633 & & & & \\
\hline$\theta_{L}$ & 0.0771 & -0.0840 & -0.0973 & 0.7127 & 0.3228 & 0.5821 & & & \\
\hline$\varphi_{L}$ & -0.0449 & 0.0526 & 0.0472 & 0.6975 & 0.9984 & 0.2855 & 2.036 & & \\
\hline$\theta_{S}$ & -0.0002 & -0.00341 & -0.0101 & -0.2898 & 0.0269 & -0.8667 & 0.0690 & 0.8522 & \\
\hline$\varphi_{S}$ & -0.0150 & 0.0194 & 0.0197 & 0.8135 & 0.9055 & 0.6809 & 0.8875 & -0.3925 & 1.3879 \\
\hline
\end{tabular}

Table 2. Correlation coefficients and errors in the estimation of parameters for a smirch corresponding to the following parameters: $m=10^{6} M_{\odot}, \eta=10^{-4}, a_{1}=0.9, \theta_{1}=\pi / 3, \varphi_{1}=$ $3 \pi / 4, \theta_{L}=\pi / 5, \varphi_{L}=\pi / 3, \theta_{S}=\pi / 2, \varphi_{S}=\pi / 3$ and $n_{\mathrm{cyc}}=9.1 \times 10^{3}$.

\begin{tabular}{|c|c|c|c|c|c|c|c|c|c|}
\hline$k$ & $m$ & $\eta$ & $a_{1}$ & $\theta_{1}$ & $\varphi_{1}$ & $\theta_{L}$ & $\varphi_{L}$ & $\theta_{S}$ & $\varphi_{S}$ \\
\hline$m$ & 0.0003 & & & & & & & & \\
\hline$\eta$ & -0.9449 & 0.0002 & & & & & & & \\
\hline$a_{1}$ & -0.9782 & 0.9358 & 0.0003 & & & & & & \\
\hline$\theta_{1}$ & 0.0057 & -0.0068 & -0.0184 & 0.5922 & & & & & \\
\hline$\varphi_{1}$ & 0.0094 & -0.0113 & -0.0091 & 0.6862 & 0.8631 & & & & \\
\hline$\theta_{L}$ & 0.0087 & -0.0160 & -0.0169 & -0.9847 & -0.7009 & 1.0201 & & & \\
\hline$\varphi_{L}$ & 0.0200 & -0.0263 & -0.0331 & 0.8773 & 0.9425 & -0.8675 & 1.2781 & & \\
\hline$\theta_{S}$ & 0.0096 & -0.0140 & 0.0014 & -0.9954 & -0.6963 & 0.9935 & -0.8761 & 1.0203 & \\
\hline$\varphi_{S}$ & -0.0082 & 0.0100 & 0.0106 & -0.1538 & 0.5862 & 0.1540 & 0.3332 & 0.1550 & 0.6145 \\
\hline
\end{tabular}

ratio is proportional to $\sqrt{\eta n_{\text {cyc }}}$ we have chosen the quantity $\sqrt{\eta n_{\text {cyc }}}$ to be roughly the same in the three examples ${ }^{3}$. For this reason, though our sources are all at the same distance, they produce similar signal-to-noise ratios. In reality they have to be integrated over different time scales, the duration being proportional to $\eta^{-1}$. Finally, note that though we have quoted a total mass (essentially the mass of the supermassive black hole) of $10^{6} M_{\odot}$ the results of this analysis hold good for other masses as well provided the mass ratios are the same. The spectral density of noise really does not vary too much over the duration of a signal so that the results discussed here are also valid for ground-based detectors with some minor modifications.

The values of the correlation coefficients whose absolute value is greater than 0.5 are shown in boldface to easily identify which parameters are correlated and which are not. A correlation coefficient of 0.5 does not mean that one of the parameters can be completely ignored. However, it does mean that the two parameters are not completely independent and

3 Indeed, as a rule of thumb all smirches with the same total mass $m$ but different $\eta(\ll 1)$, which begin at the same frequency and coalesce within the LISA band, will have the same signal-to-noise ratio. This is because, if the starting frequency is the same, then as $\eta$ is lowered by a certain factor the number of cycles, and the duration of the signal, goes up by the same factor so that $\eta n_{\mathrm{cyc}}$ is the same. Even though the duration of the signal changes quite a lot the frequency content of the signal does not change so much during this interval as a result of which the signal-to-noise ratio is roughly invariant. For ground-based sources such a rule of thumb does not apply since these sources span the entire detector band and the signal-to-noise ratio depends not only on the factor $\eta n_{\text {cyc }}$ but also on where in the frequency band those cycles are located. 
Table 3. Correlation coefficients and errors in the estimation of parameters for a smirch corresponding to the following parameters: $m=10^{6} M_{\odot}, \eta=10^{-5}, a_{1}=0.9, \theta_{1}=\pi / 4, \varphi_{1}=$ $\pi / 2, \theta_{L}=\pi / 5, \varphi_{L}=\pi / 3, \theta_{S}=\pi / 2, \varphi_{S}=\pi / 3$ and $n_{\mathrm{cyc}}=9.1 \times 10^{4}$.

\begin{tabular}{|c|c|c|c|c|c|c|c|c|c|}
\hline$k$ & $m$ & $\eta$ & $a_{1}$ & $\theta_{1}$ & $\varphi_{1}$ & $\theta_{L}$ & $\varphi_{L}$ & $\theta_{S}$ & $\varphi_{S}$ \\
\hline$m$ & $2.5 e-5$ & & & & & & & & \\
\hline$\eta$ & -0.9370 & $1.4 \mathrm{e}-5$ & & & & & & & \\
\hline$a_{1}$ & -0.8804 & 0.7873 & $2.7 e-5$ & & & & & & \\
\hline$\theta_{1}$ & -0.0315 & 0.0435 & 0.0370 & 0.4220 & & & & & \\
\hline$\varphi_{1}$ & -0.0003 & 0.0018 & -0.0182 & -0.8500 & 1.1942 & & & & \\
\hline$\theta_{L}$ & -0.0124 & 0.0188 & -0.0057 & 0.9781 & -0.8812 & 0.9363 & & & \\
\hline$\varphi_{L}$ & -0.0150 & 0.0219 & -0.0047 & -0.7517 & 0.9723 & -0.8242 & 1.1773 & & \\
\hline$\theta_{S}$ & 0.0330 & -0.0490 & -0.0097 & 0.9186 & -0.8790 & 0.9733 & -0.8637 & 0.9398 & \\
\hline$\varphi_{S}$ & 0.0040 & -0.0063 & -0.0010 & 0.1735 & 0.2853 & 0.1819 & 0.2969 & 0.1847 & 0.5546 \\
\hline
\end{tabular}

we can save some computational cost by varying a suitable combination of the two. If the correlation is close to 1 , however, we could save a lot in computational costs.

In respect of correlation coefficients the parameters fall into two categories: the angular parameters describing the orientation of the black hole's spin, orbital angular momentum and source location fall into one category and the non-angular parameters corresponding to the magnitude of the massive black hole's spin and the two masses fall into another category. The parameters within each set are correlated with one another but there is little correlation between parameters from the two categories.

Let us return to the examples given in tables $1-3$. The parameters $m, \eta$ and $s_{1}$ are so strongly correlated that only one of these three parameters suffices in a search. Similarly, looking at the correlation between the angular parameters we see that just two or three of the angular parameters might be good enough in a search. In the case of the masses and spin magnitude the correlation coefficients remain roughly the same in the three different examples. However, this is not so in the case of angular parameters. For the sake of definiteness we declare a parameter to be independent, and would therefore be needed in a search, if its correlation coefficient with the other parameters is smaller than 0.85. Comparing tables 1-3 we find that in the first example (table 1) $\theta_{1}, \varphi_{1}$, and $\theta_{L}$ are the three independent angular parameters while in the second example (table 2) $\theta_{1}, \varphi_{1}$, and $\varphi_{S}$ are the three independent parameters and in the third example $\theta_{1}$ and $\varphi_{S}$ are the independent parameters. If the independent parameters had remained the same then the search algorithm would be much simpler; we could determine forever the independent parameters and carry out a search in that space irrespective of where in the parameter space we are. However, now the independent parameters for our search (in a small region of the parameter space) depend on where in the parameter space we are. One should measure the matrix of correlation coefficients at a given point in the parameter space before embarking upon laying a grid of templates.

\section{Principal components for smirches}

Next we turn to the principal components of the spin-modulated chirp. Recollect that the principal components are nothing but the eigenvalues of the covariance matrix. In table 4 we have shown the principal components for the cases discussed in tables 1-3. We display only those components that are a significant fraction of the largest component. (A rigorous treatment of the problem should deal with dimensionless parameters so that in the process of diagonalization the transformation of the parameters is meaningful. We defer such a treatment to a later paper.) We note that the number of principal components is much less than the 
Table 4. Principal components for the three cases discussed in tables 1-3. Only components greater than $10^{-2}$ (chosen in an ad hoc manner) are listed. Cases A-C correspond to the systems discussed in tables 1-3 (see text).

\begin{tabular}{cll}
\hline Case A & Case B & Case C \\
\hline 13 & 445 & 398 \\
9.3 & 4.3 & 4.3 \\
0.19 & 0.025 & 0.11 \\
0.15 & - & 0.016 \\
0.12 & - & - \\
0.050 & - & - \\
\hline
\end{tabular}

number of parameters although the precise number of principal components depends on the point in the parameter space. Our preliminary conclusion is that the problem of catching smirches boils down to searching for three, or at most four, parameters. This means that we might need a lot fewer templates than was originally conceived. After all the problem may not really be as serious as we had originally thought.

\section{Conclusions}

Gravitational waves emitted by stellar mass black holes falling into a supermassive black hole encode a lot of information about the structure of the spacetime geometry around the massive Kerr black hole. Decoding this information requires not only accurate modelling of the emitted waveform but also algorithms that can efficiently dig out and discriminate these signals. Since the parameter space corresponding to such systems is quite large it has been thought that matched filtering, which is not only effective in detecting signals but also in deciphering their information content, would be too expensive in a search for these signals. In this paper we have explored the signal manifold corresponding to spin-modulated chirps, or smirches as we would like to call them, using the covariance matrix and the method of principal components. We conclude that the problem might be tractable after all since the effective dimensionality of the parameter space might be much smaller than what has been thought before.

The signal emitted by a small black hole in quasi-circular orbit around a massive Kerr black hole is, in general, characterized by nine search parameters - the two masses, the magnitude of the black hole's spin and its orientation, initial orientation of the orbit and the direction to the source. Our analysis has shown strong correlations between the two masses and the spin magnitude as well as between the different angular parameters. We have found strong indications that matched filtering a short duration smirch (1000 to 100000 cycles) might require a search in only three-, or at most four-, dimensional parameter space. This means that one might be able to use matched filtering to fit the strongest smirches in LISA's data and dig deeper for weaker signals. Before we can be completely optimistic about this, a Monte Carlo simulation should be performed to see if the overlap of arbitrary smirches can be larger than a certain minimal match (say 50\%) when maximized over just three parameters.

\section{Acknowledgments}

We are grateful to Sam Finn and Penn Statue University for hospitality during the LISA symposium. For support received BSS wishes to thank the Albert-Einstein Institute, Golm where this work was initiated. This research was partly supported by the Particle Physics and 
Astronomy Research Council. We benefited from useful discussions with Curt Cutler. Barak and Cutler [4] have analysed the problem independently and arrive at similar conclusions.

\section{References}

[1] Ryan F D 1995 Phys. Rev. D 525707

Finn L S and Thorne K S 2000 Phys. Rev. D 62124021

[2] Sathyaprakash B S 1998 Proc. 2nd Int. LISA Symp. ed W M Folkner (New York: AIP) p 110

[3] Apostolatos T A, Cutler C, Sussman G J and Thorne K S 1994 Phys. Rev. D 496274

Apostolatos T A 1996 Phys. Rev. D 50605

[4] Barak L and Cutler C Progress Report on Template Counting for LISA Inspiral Problem http://www.tapir.caltech.edu/listwg1/EMRI/Meetings/notes_akludge_111402.ps 